\title{
Optimizing spatial pore-size and porosity distributions of adsorbents for enhanced adsorption and desorption performance
}

\author{
Guanghua Ye ${ }^{\mathrm{a}, \mathrm{b}}$, Xuezhi Duan ${ }^{\mathrm{a}}$, Kake Zhu ${ }^{\mathrm{a}}$, Xinggui Zhou ${ }^{\mathrm{a}, *}$, Marc-Olivier Coppens ${ }^{\mathrm{b}}$, \\ Weikang Yuan ${ }^{a}$
}

a State Key Laboratory of Chemical Engineering, East China University of Science and Technology, Shanghai 200237, China

${ }^{\mathrm{b}}$ Department of Chemical Engineering, University College London, London WC1E 7JE, UK

\section{H I G H L I G H T S}

- A method is proposed to optimize the spatial structure of adsorbents.

- A uniform spatial structure is proven to be the preferred one.

- The average porosity of adsorbents is optimized.

- The method is relevant to PSA processes, where the effect of the adsorption/desorption cycle period is considered.

\section{A R T I C L E I N F O}

\section{Article history:}

Received 23 February 2015

Received in revised form

10 April 2015

Accepted 11 April 2015

Available online 28 April 2015

Keywords:

Spatial distributions

Adsorbent design

Mass transfer

Continuum approach

Adsorption/desorption performance

Optimal porosity

\begin{abstract}
A B S T R A C T
This paper shows that a uniform spatial distribution in meso/macroporosity of adsorbents maximizes their adsorption and desorption performance. It highlights the importance of optimizing porosity and pore diameter, not only at the nanoscale but also at larger length scales. The effects of spatial pore size and porosity distributions on mass transfer in adsorbents are studied by using a continuum approach. These effects are evaluated by comparing the adsorption/desorption performance of adsorbents subjected to a square wave concentration perturbation with a wide range of cycle period $(10-100,000 \mathrm{~s})$ for the adsorption of $\mathrm{n}$-pentane on $5 \mathrm{~A}$ zeolite adsorbents. The uniformly distributed pore size and porosity is the preferred structure, which is confirmed by using four empirical tortuosityporosity relations. Further optimization of the uniform structure shows that its optimal average porosity is in the range of 0.4-0.6 when the perturbation cycle period is between $100 \mathrm{~s}$ and $2000 \mathrm{~s}$ and the volume-averaged pore diameter is between $10 \mathrm{~nm}$ and $150 \mathrm{~nm}$. The relationships between optimal average porosity, cycle period and volume-averaged pore diameter are determined and explained. These results should serve to guide the synthesis of adsorbents.
\end{abstract}

(c) 2015 Elsevier Ltd. All rights reserved.

\section{Introduction}

Studying mass transfer in porous media is important for designing industrial adsorbents. Most of the past studies have focused on the effects of statistical parameters (e.g., pore size distribution, average porosity and average connectivity) on mass transfer. But only few of these studies have investigated the effects of spatial structures, such as the spatial distributions of pore size and porosity. Given the great progress in synthesizing porous media with a controlled structure (Yao et al., 2010; Triantafillidis et al., 2013; Petkovich and Stein, 2013), knowledge about the

\footnotetext{
* Corresponding author. Tel.: +8621 64253509; fax: +862164253528.

E-mail address: xgzhou@ecust.edu.cn (X. Zhou).
}

effects of spatial structures on mass transfer in porous media is increasingly relevant for the design of industrial adsorbents.

The effects of spatial structures on mass transfer in catalysts have received attention by Coppens and his coworkers (Gheorghiu and Coppens, 2004; Johannessen et al., 2007; Wang et al., 2007; Wang and Coppens, 2008, 2010; Rao and Coppens, 2010, 2012), who investigated how the spatially distributed mesopore or macropore size and porosity in nanoporous catalysts affect catalyst performance. Their findings revealed that catalysts with uniformly distributed mesopores or macropores between nanoporous grains of optimized size are close to optimal. In their work, a continuum modeling approach was employed to optimize the pore positions, pore sizes, pore density and pore network geometry. The continuum approach is simple, efficient and appropriate for the structural optimization of pore networks sufficiently far removed from the percolation threshold and for reactions that are not nearly instantaneous (Zhang and 
Seaton, 1992). For a single reaction, the generalized Thiele Modulus method is useful, because the simple relationship between Thiele Modulus and effectiveness factor provides direct insight to understand which structure is optimal.

However, in adsorption studies, no systematic study on the effects of spatial distributions of pore size and porosity on mass transfer in adsorbents has been reported. To bridge this gap, a proper modeling approach and a reasonable evaluation method should be established first.

Continuum and discrete modeling approaches are available to model mass transfer in adsorbents. These have been reviewed by Sahimi et al. (1990) and Keil (1999). Continuum approaches include the parallel pore model (Wheeler, 1951), tortuous pore model (Carman, 1937), cylindrical pore model (Johnson and Stewart, 1965), model of Wakao and Smith (1962, 1964), model of Foster and Butt (1966), micro/macropore model (Mann and Thomson, 1987) and grain model (Szekely and Evans, 1970). The continuum approach is efficient and may be appropriate for structural optimization, however the heterogeneity at length scales comparable to individual pores is neglected in structural optimization (Wang et al., 2007). Recently, a digitally reconstructed pore model has been introduced in combination with the continuum approach (Kočí et al., 2006, 2010; Novák et al., 2010, 2013, 2014; Václavík et al., 2014; Seda et al., 2008). Applying this pore model, the heterogeneity at different length scales can be included in simulations, however it is still difficult to apply for the purpose of optimizing the pore structure of porous media, because the computational cost is very high.

When using a discrete modeling approach, the Bethe lattice (Beeckmann and Froment, 1980), Voronoi grids (Voronoi, 1908; Winterfeld et al., 1981) and various other pore network models (Hollewand and Gladden, 1992) are widely employed (Sahimi et al., 1990; Keil, 1999). Particularly, random pore network models are advantageous in accounting for the spatially distributed properties of the pore network structure of porous media (Keil, 1999). Nevertheless, the computational cost of structural optimizations is high, especially when involving time-dependent dynamic simulations of mass transfer in adsorbents, as in this work.

To evaluate the adsorption/desorption performance of adsorbents with specific spatial structures, a proper evaluation criterion must be defined. Rezaei and Webley (2012) proposed using the working capacity of a single adsorbent subjected to a square wave concentration perturbation. This working capacity defines the difference between the adsorbed amount at the end of the adsorption period and desorption period when the cyclic adsorption process is in the cyclic steady state (CSS), as seen in Fig. 2b. The working capacity (non-equilibrium) accounts for both the adsorption capacity and adsorption kinetics of adsorbents, as well as the effects of transport limitations that come into play when adsorption or desorption equilibrium is not reached. Therefore, the working capacity is more informative than the effective diffusivity by itself in evaluating the effects of spatial structures on the adsorption/desorption performance.

Based on the above considerations, a continuum approach is used to probe the effects of three representative spatial distributions of pore size and porosity on mass transfer in adsorbents. The working capacity of a single adsorbent under a square wave perturbation with a wide range of cycle period is employed to quantify the adsorption/desorption performance of adsorbents with designated spatial structures. The three characteristic spatial distributions are studied in detail: uniform spatial distribution of pore size and porosity (D1); pore size and porosity descending toward the center of spherical adsorbents (D2); and pore size and porosity ascending toward the center of spherical adsorbents (D3). The preferred spatial structure is determined by comparing the working capacities of the adsorbents with the three characteristic spatial structures (i.e., D1, D2 and D3), and then further substantiated by comparing with 1000 other random spatial structures and using four empirical tortuosity-porosity relations substituted in the mass transfer equations. Finally, the average porosity of adsorbents with the preferred spatial structure is optimized under different perturbation cycle periods and volume-averaged pore diameters.

\section{Modeling approach}

\subsection{Modeling assumptions}

In this work, spherical adsorbent particles are assumed to consist of nanoporous microparticles (e.g., zeolite crystals). The large-scale pore structure of the adsorbents is characterized by the spatial distributions of mesopores and macropores between microparticles, which are reflected in spatial distributions of pore size and porosity. When the microparticles themselves are large, the mass balance equations for micropores should be coupled with those for large pores (Ye et al., 2014). The concentration gradients in microparticles could weaken but will not qualitatively change the effects of the spatial distributions of large pores between microparticles, similar to the results of Ye et al. (2014) for evaluating different approximations of micropore diffusion in bidisperse adsorbents. Furthermore, for catalytic problems, it was previously demonstrated (Johannessen et al., 2007; Wang et al., 2007) that the global optimum corresponds to one where there are no diffusion limitations in the nanoporous grains or microparticles. Therefore, only the effects of spatial distributions of large pores are studied in this work.

Mass transfer in porous media generally involves diffusion and convection. In wider macropores convective flow might be important and should be considered as well (Wheeler, 1951; Rodrigues et al., 1982, 1991; Carta and Rodrigues, 1993; Lu et al., 1993), while, in narrow pores at high pressures, surface diffusion may play a significant role. However, these effects are neglected, as the mass transfer rate is typically governed by molecular diffusion and Knudsen diffusion in the range of pore sizes considered here (mesopores and narrow macropores). This assumption is reasonable in a number of cases, as corroborated by experiments (Silva and Rodrigues, 1997a; Da Silva and Rodrigues, 1999; Bárcia et al., 2005).

Additional assumptions are that there are no temperature gradients in the adsorbents (Solsvik and Jakobsen, 2011), and there is instantaneous local equilibrium between the gas phase and the adsorbed phase.

\subsection{Pore structure model for the adsorbent}

The model describing the large pore structure of adsorbents is similar to that of catalysts in the work of Rao and Coppens (2012), in that a spherical porous particle is divided into a number of concentric zones characterized by tunable pore structural parameters, as illustrated in Fig. 1. The two models also have a significant difference. This model is based on the random-pore model of Wakao and Smith $(1962,1964)$, where the pore size and porosity can be involved directly and separately in mass transfer equations. However, the model of Rao and Coppens is based on the random spheres model (Weissberg, 1963), where the pore size and porosity are functions of the size of randomly distributed spheres and their number density.

When only investigating the effects of spatial distributions of pore size on mass transfer, the porosity is assigned to be $\varepsilon_{a}$, i.e., 0.5 (see Table 1), for all the zones. The statistical pore volume distribution (PVD) is assumed to be based on a Gaussian 
distribution for the pore diameter, $d$ :

$f(d)=\frac{1}{\sigma \sqrt{2 \pi}} \exp \left[-\left(\frac{d-d_{a}}{\sqrt{2} \sigma}\right)^{2}\right]$

where $d_{a}$ is the volume-averaged pore diameter and $\sigma$ is the standard deviation. A lower limit and an upper limit of pore diameter are deliberately assigned to avoid undersized or oversized pores. The lower limit of the pore diameter $\left(d_{L}\right)$ is $2 \mathrm{~nm}$, which is the lower boundary of mesopore size according to IUPAC. The upper limit of the pore diameter $\left(d_{H}\right)$ is set to $150 \mathrm{~nm}$ to cover the whole range of mesopore sizes $(2-50 \mathrm{~nm})$ and a range of macropore sizes. Thus, a modified Gaussian distribution is employed:

$F(d)=\frac{\frac{1}{\sigma \sqrt{2 \pi}} \exp \left[-\left(\frac{d-d_{a}}{\sqrt{2} \sigma}\right)^{2}\right]}{\int_{d_{L}}^{d_{H}} \frac{1}{\sigma \sqrt{2 \pi}} \exp \left[-\left(\frac{d-d_{a}}{\sqrt{2} \sigma}\right)^{2}\right] \mathrm{d} d} \quad d_{L} \leq d \leq d_{H}$

For the structures D1, D2 and D3, with the predefined porosity ( $\varepsilon_{n}=0.5$, for all zones), the pore diameter, $d_{n}$, of the ' $n$ th' zone $(n=1,2, \ldots, N)$ can be implicitly calculated according to Eqs. (3), (4) and (5), respectively. The numerical solutions are presented in the inset of Fig. 3.

$d_{n}=\int_{d_{L}}^{d_{H}}[d F(d)] \mathrm{d} d$

$\int_{d_{L}}^{d_{n}}[F(d)] \mathrm{d} d=\frac{\sum_{i=1}^{n} V}{V_{p}}=\frac{R_{n}^{3}}{R_{p}^{3}}$

$\int_{d_{n}}^{d_{H}}[F(d)] \mathrm{d} d=\frac{\sum_{i=1}^{n} V}{V_{p}}=\frac{R_{n}^{3}}{R_{p}^{3}}$

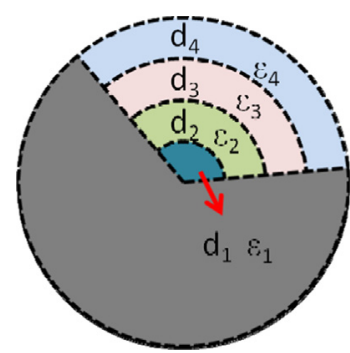

Fig. 1. $N$-zone adsorbent with tunable pore diameter and porosity in each zone. In this illustration, $N=4$.
In Eqs. (4) and (5), $V_{i}$ and $R_{n}$ are the pore volume and the radial position of the corresponding zone, while $V_{p}$ and $R_{p}$ are the pore volume and the radius of the adsorbent pellets, respectively.

When only investigating the effects of spatial distributions of porosity on mass transfer, the pore diameter is set to $d_{a}$, i.e., $76 \mathrm{~nm}$ (see Table 1), for all the zones. The porosity is assumed to be constant within each zone. With the predefined pore diameter $\left(d_{n}=76 \mathrm{~nm}\right.$, for all zones), the porosity, $\varepsilon_{n}$, of the ' $n$ th' zone ( $n=1$, $2, \ldots, N)$ of structure D1, D2 or D3 is determined according to Eqs. (6), (7) and (8), respectively.

$\varepsilon_{n}=\frac{\varepsilon_{H}+\varepsilon_{L}}{2}$

$\varepsilon_{n}=\varepsilon_{L}+\left(\varepsilon_{H}-\varepsilon_{L}\right) \frac{R_{n}^{3}}{R_{p}^{3}}$

$\varepsilon_{n}=\varepsilon_{H}-\left(\varepsilon_{H}-\varepsilon_{L}\right) \frac{R_{n}^{3}}{R_{p}^{3}}$

where $\varepsilon_{L}$ and $\varepsilon_{H}$ are the low limit and the high limit of porosity, respectively. To cover a wide range of porosity, $\varepsilon_{L}$ and $\varepsilon_{H}$ are assigned to be 0.1 and 0.9 , respectively.

\subsection{Mass transfer equations}

Adsorption of a single component in a binary mixture is considered in this study, so that Fick's law is applied to describe mass transfer in the adsorbents, rather than the more complex Stefan-Maxwell equations (Krishna and Wesselingh, 1997):

$N_{i}=-D_{e, i} \frac{\mathrm{d} C_{i}}{\mathrm{~d} R}$

where $N_{i}$ is the flux of component $i$ and $C_{i}$ is the concentration of component $i$ in the mesopores or macropores. The overall effective diffusivity $\left(D_{e, i}\right)$ of component $i$ combines the binary molecular diffusivity $\left(D_{i, j}\right)$ of component $i$ in a mixture of $i$ and $j$, and the Knudsen diffusivity $\left(D_{k, i}\right)$ of component $i$, and is calculated using Bosanquet's approximation:

$D_{e, i}=\frac{\varepsilon}{\tau} \frac{D_{k, i} D_{i, j}}{D_{k, i}+D_{i, j}}$

where $\tau$, tortuosity, is approximated by $1 / \varepsilon$, using Wakao and Smith's (1962, 1964) model. The latter assumption is not essential for the main conclusions; other $\tau(\varepsilon)$ relations will be considered further on Using $\tau(\varepsilon)=1 / \varepsilon$, the effective diffusivity of component $i$ is

$D_{e, i}=\varepsilon^{2} \frac{D_{k, i} D_{i, j}}{D_{k, i}+D_{i, j}}$

Table 1

Parameters for the cyclic adsorption/desorption simulations

\begin{tabular}{|c|c|c|c|}
\hline Property & Symbol & Unit & Value \\
\hline High limit of the pore diameter & $d_{H}$ & $\mathrm{~nm}$ & 150 \\
\hline Low limit of the pore diameter & $d_{L}$ & $\mathrm{~nm}$ & 2 \\
\hline Volume-averaged pore diameter & $d_{a}$ & $\mathrm{~nm}$ & 76 \\
\hline Standard deviation & $\sigma$ & $\mathrm{nm}$ & 40 \\
\hline High limit of the porosity & $\varepsilon_{H}$ & - & 0.90 \\
\hline Low limit of the porosity & $\varepsilon_{L}$ & - & 0.10 \\
\hline Average porosity & $\varepsilon_{a}$ & - & 0.50 \\
\hline Pellet radius & $R_{p}$ & $\mathrm{~m}$ & $1.55 \times 10^{-3}$ \\
\hline Henry coefficient for n-pentane adsorbed in 5A zeolite (Silva and Rodrigues, 1997b) & $K$ & - & 3031.90 \\
\hline Temperature (Silva and Rodrigues, 1997b) & $T$ & $\mathrm{~K}$ & 473 \\
\hline Total pressure & $P$ & bar & 1 \\
\hline Perturbation cycle period & $\theta$ & s & $10-100,000$ \\
\hline Maximum bulk concentration & $C_{\max , i}$ & $\mathrm{~mol} / \mathrm{m}^{3}$ & 1 \\
\hline Minimum bulk concentration & $C_{\min , i}$ & $\mathrm{~mol} / \mathrm{m}^{3}$ & 0 \\
\hline
\end{tabular}


a

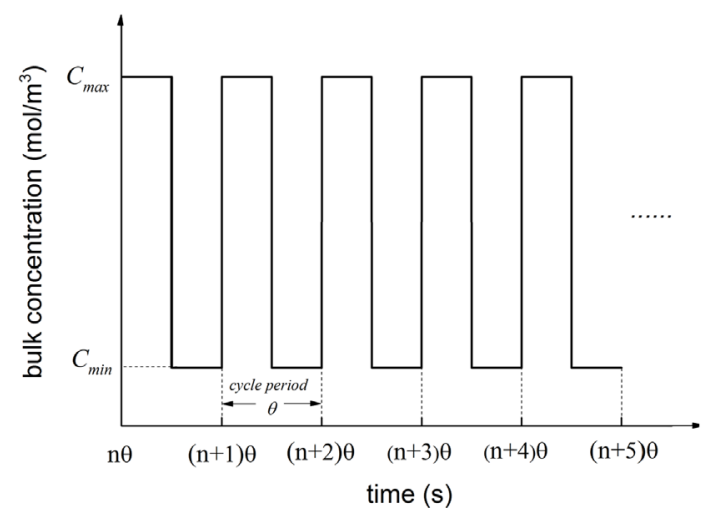

b

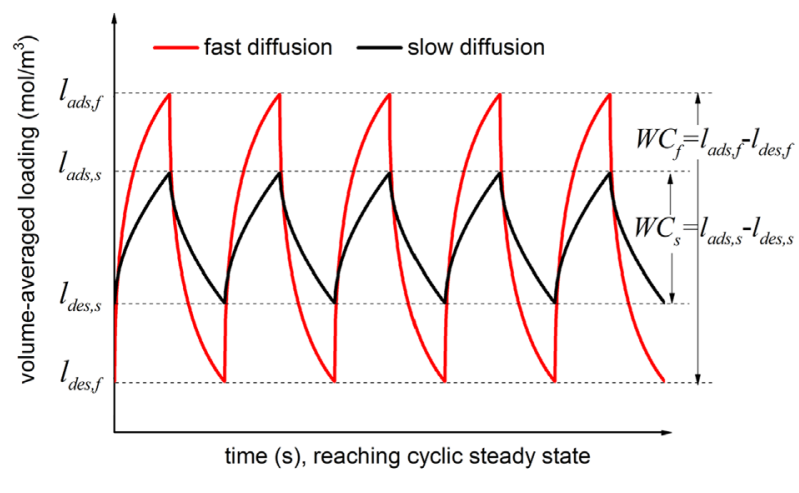

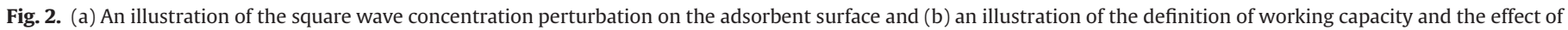
diffusion in the adsorbent on working capacity.

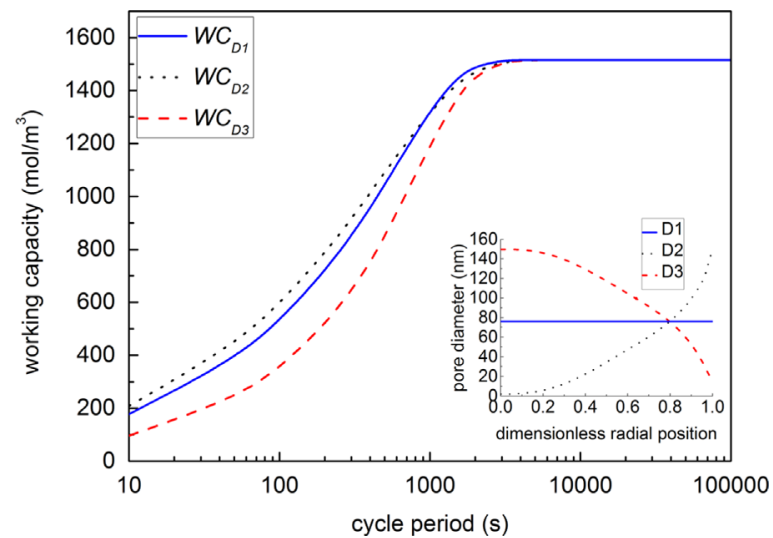

Fig. 3. Working capacities as a function of the perturbation cycle period for the three archetypical spatial distributions of pore size, shown in the inset.

Both pore size and porosity affect mass transfer in adsorbents through changes in effective diffusivity.

The binary molecular diffusivity of component $i$ in a mixture of $i$ and $j$ is calculated by using the Chapman-Enskog equation (Poling et al., 2001):

$$
D_{i, j}=\frac{1.8809 \times 10^{-7} \sqrt{T^{3}\left(\frac{1}{M_{i}}+\frac{1}{M_{j}}\right)}}{P \sigma_{i, j}^{2} \Omega_{i, j}},
$$

$\Omega_{i, j}=1.06036\left(\frac{\varepsilon_{i, j}}{k T}\right)^{0.15610}+0.19300 e^{-0.47635 \frac{k T}{\varepsilon_{i, j}}}+1.03587 e^{-1.52996 \frac{k T}{\varepsilon_{i, j}}}$

$$
+1.76474 e^{-3.89411 \frac{k T}{\varepsilon_{i, j}}}
$$

$\sigma_{i, j}=\frac{\left(\sigma_{i}+\sigma_{j}\right)}{2}$

$\varepsilon_{i, j}=\sqrt{\varepsilon_{i} \varepsilon_{j}}$,

where $\sigma_{i}$ and $\varepsilon_{i} / k$ are the characteristic Lennard-Jones length and energy of component $i$. The Knudsen diffusivity of component $i$ is computed from the kinetic theory of gases (Welty et al., 2008):

$D_{k, i}=10^{-9} \times \frac{d}{3} \sqrt{\frac{8 \times 10^{3} R_{g} T}{\pi M_{i}}}$.

\subsection{Mass balance equations}

The change over time of the concentration, $C_{i}$, of adsorbate $i$ in the mesopores or macropores is described by the following partial differential equation (Ruthven, 1984):

$\varepsilon \frac{\partial C_{i}}{\partial t}+(1-\varepsilon) \frac{\partial q_{i}}{\partial t}=-\frac{1}{R^{2}} \frac{\partial}{\partial R}\left(R^{2} N_{i}\right)$

with the following boundary and initial conditions:

$\frac{\partial C_{i}}{\partial R}=0 \quad$ at $\quad R=0, \quad t>0$

$C_{i}=C_{b, i} \quad$ at $\quad R=R_{p}, \quad t>0$

$C_{i}=0 \quad$ at $\quad t=0, \quad 0 \leq R \leq R_{p}$

where $N_{i}$ is represented by Eq. (9). The loading of adsorbate $i$ in the microparticles, $q_{i}$, is assumed to follow Henry's law, i.e.,

$q_{i}=K C_{i}$

when the concentration of adsorbate $i, C_{i}$, is low. However, when $C_{i}$ is high, a nonlinear isotherm should be used. Similar to the results of Coppens and co-workers for non-linear single-reaction kinetics (Wang and Coppens, 2010), the results in this work could be quantitatively different but still qualitatively valid, because only the effective diffusivity is different among different spatial structures. In Eq. (14c), $C_{b, i}$ is the bulk concentration of adsorbate $i$, which fluctuates in time according to the square wave concentration perturbation as

$C_{b, i}=\left\{\begin{array}{cc}C_{\max , i} & n \theta \leq t<(2 n+1) \theta / 2 \\ C_{\min , i} & (2 n+1) \theta / 2 \leq t<(n+1) \theta\end{array} \quad n=0,1,2,3 \ldots\right.$

where $C_{\max , i}$ and $C_{\min , i}$ are the maximum and minimum bulk concentration of component $i$, respectively and $\theta$ is the perturbation cycle period. The values of $C_{\max , i}$ and $C_{\min , i}$ do not affect the comparison among different spatial structures if the assumption of Henry's law is reasonable; the latter should of course always be verified. Fig. 2a shows the square wave concentration perturbation on the adsorbent surface. Finally, substituting Eqs. (15) and (9) into Eq. (14a), one has

$[\varepsilon+(1-\varepsilon) K] \frac{\partial C_{i}}{\partial t}=\frac{1}{R^{2}} \frac{\partial}{\partial R}\left(R^{2} D_{e, i} \frac{\partial C_{i}}{\partial R}\right)$.

\subsection{Working capacity}

The adsorption/desorption performance of the adsorbents with different spatial structures is quantified by the working capacity, defined as (Rezaei and Webley, 2012)

$W C=l_{\text {ads }, i}-l_{\text {des }, i}$

where $l_{a d s, i}$ and $l_{d e s, i}$ are the volume-averaged loading of component $i$ at the end of the adsorption period and the desorption period, respectively, after reaching the cyclic steady state (CSS). 
The volume-averaged loading of adsorbate $i\left(l_{i}\right)$ is defined as

$l_{i}=\frac{\int_{0}^{R_{p}}[\varepsilon+(1-\varepsilon) K] C_{i} 4 \pi R^{2} d R}{\frac{4}{3} \pi R_{p}^{3}}$

Fig. 2b illustrates the working capacity. In this article, the working capacity (non-equilibrium) reflects both adsorption capacity and adsorption kinetics of adsorbents. If the amount of adsorbing material is constant, the working capacity (non-equilibrium) is higher with faster diffusion in adsorbents.

\subsection{Numerical method and simulation parameters}

The simulations are performed by employing MATLAB R2014a, where Eq. (17) is discretized by using the orthogonal collocation method. Table 1 summarizes the parameters for simulating the cyclic adsorption/desorption of n-pentane in nitrogen on industrial $5 \mathrm{~A}$ zeolite adsorbents that are inactive to nitrogen.

\section{Results and discussion}

\subsection{Effects of spatial pore-size distribution}

In this section, the effects of the three archetypical spatial distributions (i.e., D1, D2 and D3) of pore size on mass transfer and the associated working capacities are investigated, while the porosity in every zone of the adsorbents is assumed to be constant ( $\varepsilon_{n}=0.5$, for all zones). Fig. 3 compares the three working capacities (i.e., $W C_{D 1}, W C_{D 2}$ and $W C_{D 3}$ ) of the adsorbents when the perturbation cycle period is changed from $10 \mathrm{~s}$ to $100,000 \mathrm{~s}$.

$W C_{D 1}, W C_{D 2}$ and $W C_{D 3}$ all increase with increasing cycle period before reaching the same maximum working capacity $\left(1516.45 \mathrm{~mol} / \mathrm{m}^{3}\right)$. When extending the cycle period, the adsorbed amount at the end of the adsorption period $\left(l_{a d s, i}\right)$ increases until it reaches the equilibrium value $\left(1516.45 \mathrm{~mol} / \mathrm{m}^{3}\right)$, while the adsorbed amount at the end of the desorption period $\left(l_{d e s, i}\right)$ decreases until it reaches $0 \mathrm{~mol} / \mathrm{m}^{3}$. According to the definition of working capacity (Eq. (18)), the three working capacities ultimately ascend to the same value, because the average porosity of all adsorbents is the same $\left(\varepsilon_{a}=0.5\right)$.

$W C_{D 1}$ is largest when the cycle period is over $1000 \mathrm{~s}$, while $W C_{D 2}$ is largest when the cycle period is below $1000 \mathrm{~s}$. Practically, the uniformly distributed (D1) pore size is the preferred structure in terms of designing adsorbents, because the uniform structure is easier to make and the difference between $W C_{D 1}$ and $W C_{D 2}$ is slight (no more than $4.5 \%$ of the equilibrium value). Coppens and his coworkers (Gheorghiu and Coppens, 2004; Johannessen et al., 2007; Wang et al., 2007; Wang and Coppens, 2008; Rao and Coppens, 2010, 2012) similarly found that catalysts with uniformly distributed (D1) pore size and porosity operate very near optimality.

To explain the results presented in Fig. 3, the loading profiles in the adsorbent at different operation times in one adsorption/desorption cycle are presented in Fig. 4. The different spatial distributions of pore size cause distinct distributions of diffusional resistance, resulting in different shapes of the loading profile. Therefore, we can directly perceive the effects of the spatial distributions of pore size on mass transfer in adsorbents by observing the loading profiles.

As seen from Fig. 4b and e, for the adsorbent with pore size distribution D2, the concentrations around the adsorbent center $\left(R^{*}=0\right)$ change slightly with time, indicating the extremely low flux of adsorbate in the center zones. The low flux would deteriorate the overall adsorption/desorption performance of the adsorbents. Fig. 4b and e also shows that the concentration gradients in the outer zones are not as steep as those shown in Fig. 4a and $d$ for the uniform distribution (D1) of the pore size, implying a smaller diffusional resistance in outer zones corresponding to broader pores in those zones. Such a small diffusional resistance would improve the overall adsorption/desorption performance of adsorbents, especially under a short cycle period. The above two competing factors determine whether $W C_{D 2}$ is larger than $W C_{D 1}$ or not.

As seen from Fig. 4c and f, for the adsorbent with pore size distribution D3, the concentration gradients in outer zones are steeper than the corresponding ones shown in Fig. 4a and d, implying a larger diffusional resistance in the outer zones where the pores are narrower. The outer zone is crucial to mass transfer in adsorbents, because it is exposed to the environment. Hence, the adsorption/desorption performance of adsorbents would be significantly limited even by a limited increase on diffusional resistance in the outer zones.

\subsection{Effects of spatial porosity distribution}

In this section, only the effects of the three archetypical spatial distributions (i.e., D1, D2 and D3) of porosity are studied, while the pore diameter in every zone of the adsorbents is assumed to be constant ( $d_{n}=76 \mathrm{~nm}$, for all zones). Fig. 5 shows the corresponding three working capacities (i.e., $W C_{D 1}, W C_{D 2}$ and $W C_{D 3}$ ) as a function of the perturbation cycle period.

$W C_{D 1}, W C_{D 2}$ and $W C_{D 3}$ all increase to the same maximum $\left(1516.45 \mathrm{~mol} / \mathrm{m}^{3}\right)$, which coincides with the result presented in Section 3.1 and can be explained in the same way. $W C_{D 1}$ is largest, although the difference between $W C_{D 1}$ and $W C_{D 2}$ decreases with the decreasing cycle period. Therefore, a uniform porosity (D1) is preferred in designing adsorbents. Similar results were also reported in catalysis (Gheorghiu and Coppens, 2004; Johannessen et al., 2007; Wang et al. 2007; Wang and Coppens, 2008; Rao and Coppens, 2010, 2012).

Fig. 6 presents the loading profiles in the adsorbents with different spatial porosity distributions and at different operation times in one adsorption/desorption cycle. As shown in Fig. 6b and e, for the adsorbent with spatial porosity distribution D2, the concentrations in the center zones remain nearly unchanged, indicating that the inner part (around $6.4 \%$ of the total amount of adsorbing material, according to Fig. $6 \mathrm{~b}$ and e) of the adsorbent remains virtually unchanged. Although the small diffusional resistance in the outer zones would improve the adsorption/desorption performance of adsorbents to some extent, near-absence of any flux in the inner zones deteriorates the adsorption/desorption performance of these adsorbents significantly.

As seen from Fig. $6 \mathrm{c}$ and $\mathrm{f}$, for the adsorbent with porosity distribution D3, the concentration gradients in the outer zones are much steeper than the corresponding ones shown in Fig. 6a and d. Obviously, mass transfer in the adsorbent with distribution D3 of porosity is strongly limited due to the low porosity in the outer shell, resulting in the poor adsorption/desorption performance of such adsorbents.

\subsection{Further validation of the preferred spatial structure}

To further substantiate that the uniform structure (D1) is preferred, the structure D1 is compared with the optimal spatial structure that has the largest working capacity among a number of randomly generated spatial structures, as illustrated in Fig. 7. The randomly generated spatial structures are given the same volumeaveraged pore diameter ( $76 \mathrm{~nm}$ ) and average porosity (0.5) to ensure that the spatial distribution is the only variable affecting mass transfer in the adsorbents. Only a negligible difference in optimal working capacity is found when increasing the number of Monte-Carlo generated spatial structures from 1000 to 2000. Moreover, the working capacity is sensitive to spatial structures when the perturbation cycle period is between $100 \mathrm{~s}$ and $2000 \mathrm{~s}$ (see Figs. 3 and 5). Therefore, we randomly generate 1000 spatial 

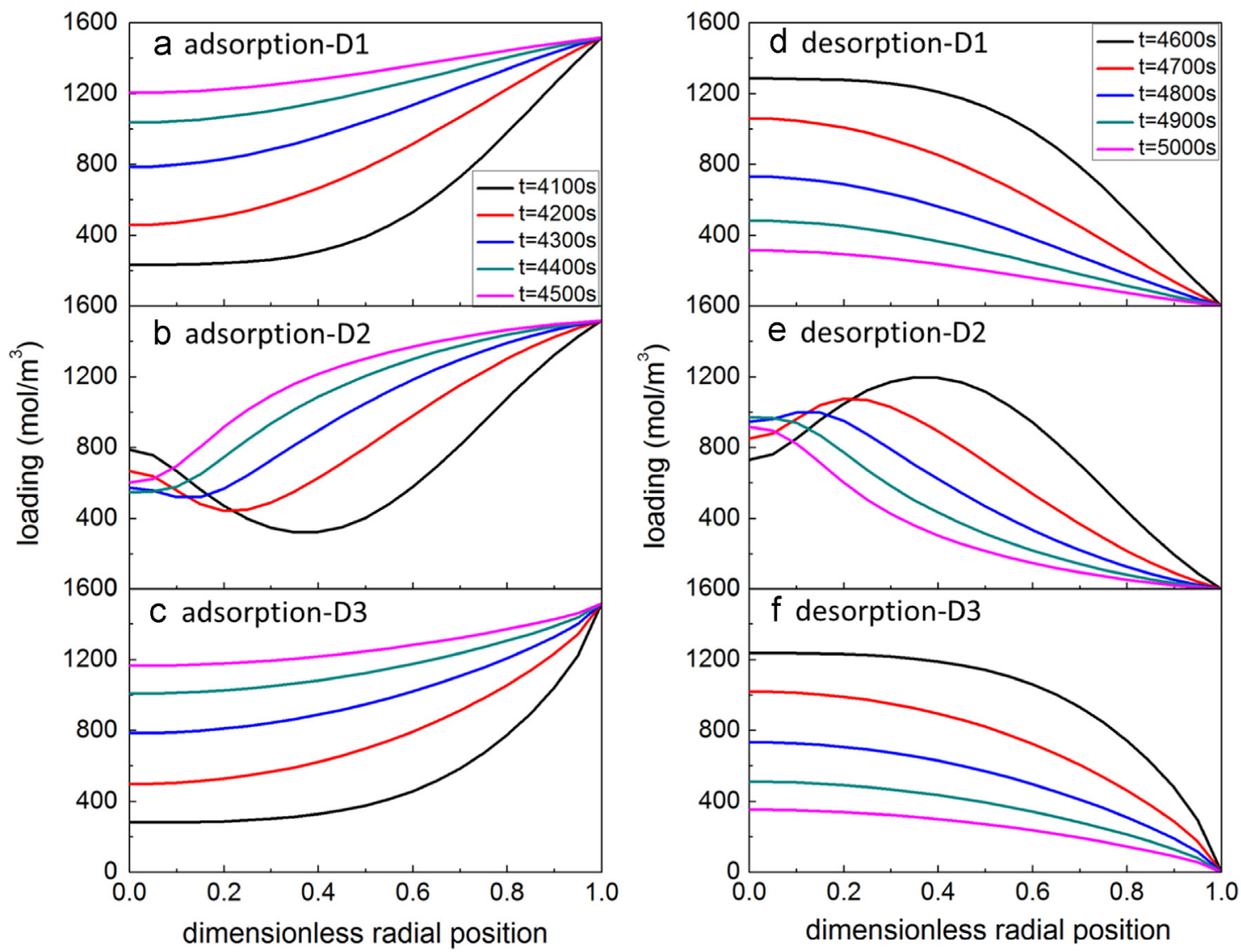

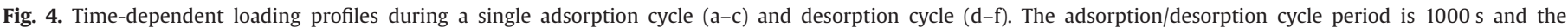
adsorption/desorption reaches the cyclic steady state in its 5th cycle. D1, D2 and D3 represent different spatial distributions of pore size, as shown in Fig. 3.

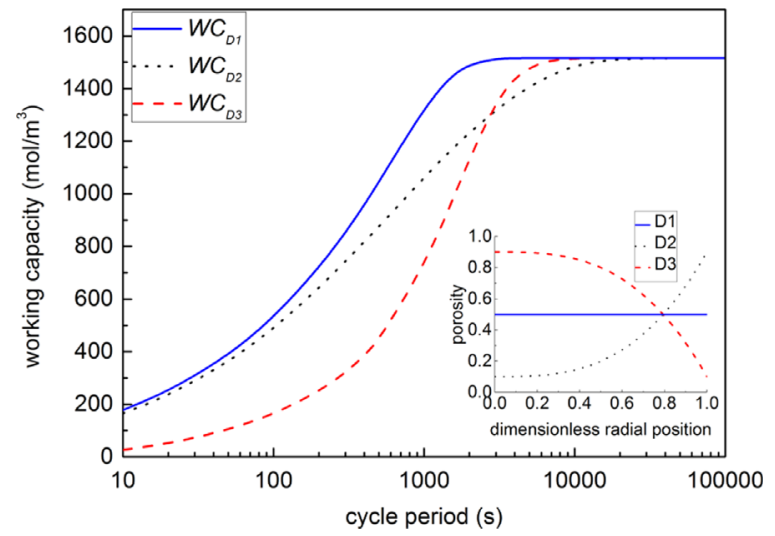

Fig. 5. Working capacities as a function of the perturbation cycle period for the three archetypical spatial distributions of porosity, shown in the inset.

structures in this comparison study, while varying the cycle period from $100 \mathrm{~s}$ to $2000 \mathrm{~s}$.

As seen from Fig. $7, W C_{D 1}$ is very close to $W C_{\text {optimal }}$ amongst these randomly generated structures, and the largest difference between $W C_{D 1}$ and $W C_{\text {optimal }}$ is below $7 \%$ of the equilibrium value $(1516.45 \mathrm{~mol} /$ $\mathrm{m}^{3}$ ). For cycle periods above $1000 \mathrm{~s}$, there is no significant difference between $W C_{D 1}$ and $W C_{\text {optimal }}$. From a practical point of view, the uniform structure (D1) thus remains the preferred structure, as it has a similar performance, and is easier to produce than any of the optimal structures amongst the Monte-Carlo generated samples.
The spatial distributions in Fig. 7 for the samples with maximum performance fluctuate wildly in the inner zones of the spherical adsorbents, because the working capacity is numerically insensitive to large variations in spatial distribution in the core zones. The number of randomly generated spatial structures is also not large enough to detect a structure that might be marginally better. However, the optimal spatial distributions fluctuate much less in the outer zones, because these are where most of the adsorption and desorption occur, so that the corresponding working capacity is highly sensitive to the spatial distribution. As noted earlier, the descending distribution of pore size in the outer zones is optimal when the cycle period is quite short (e.g., $100 \mathrm{~s}$ ), so that $W C_{\text {optimal }} \approx W C_{D 2}$, while a uniform distribution of porosity in the outer zones is optimal when the cycle period is long enough (e.g., $1000 \mathrm{~s}$ ), so that $W C_{\text {optimal }} \approx W C_{D 1}$. These results are consistent with the results presented in Sections 3.1 and 3.2. More details on the optimal spatial structure are given in Supporting information.

In addition, the effects of different tortuosity-porosity relations, $\tau(\varepsilon)$, on the working capacities of the uniform structure (D1) and the optimal structure are probed, as shown in Fig. 8, for a 'worse case scenario' with short cycle period. Four frequently used tortuosity-porosity relations are included. They are $\tau=1-0.5 \ln \varepsilon$ (Weissberg, 1963), $\tau=1 / \varepsilon^{0.5}$ (Bruggeman, 1935), $\tau=1 / \varepsilon$ (Wakao and Smith, 1962, 1964) and $\tau=\sqrt{3} / \varepsilon$ (Weisz and Schwartz, 1962). Working capacities are different when using different tortuosityporosity relations, because tortuosity affects the effective diffusivity according to Eq. (10). However, the difference between the working capacities of the uniform structure and that of the optimal spatial structures is still small and only slightly changed. 

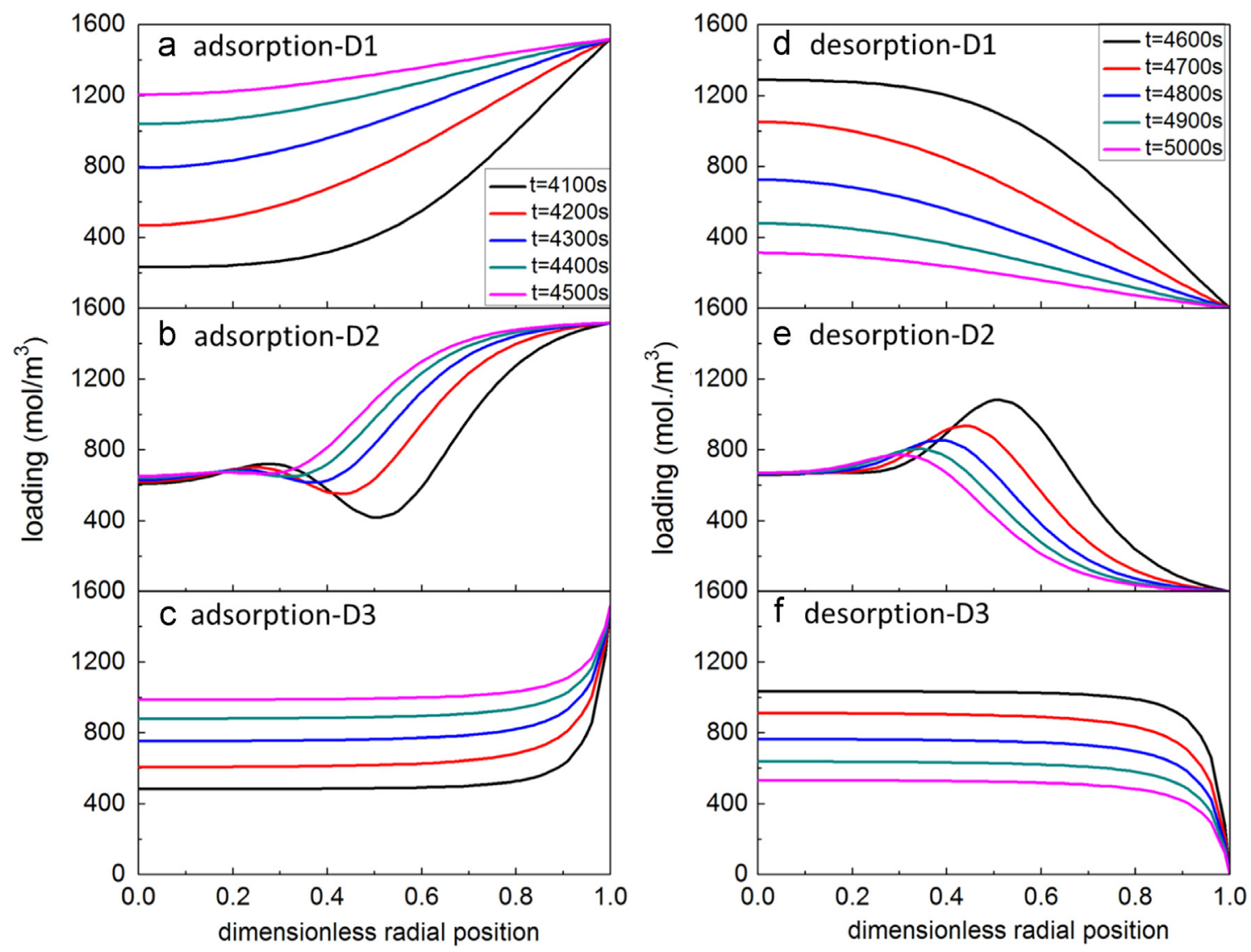

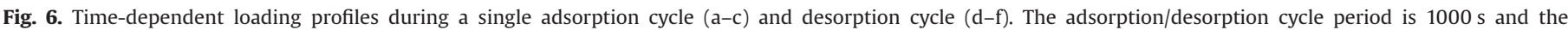
adsorption/desorption reaches the cyclic steady state in its 5th cycle. D1, D2 and D3 represent different spatial distributions of porosity, as shown in Fig. 5.

a

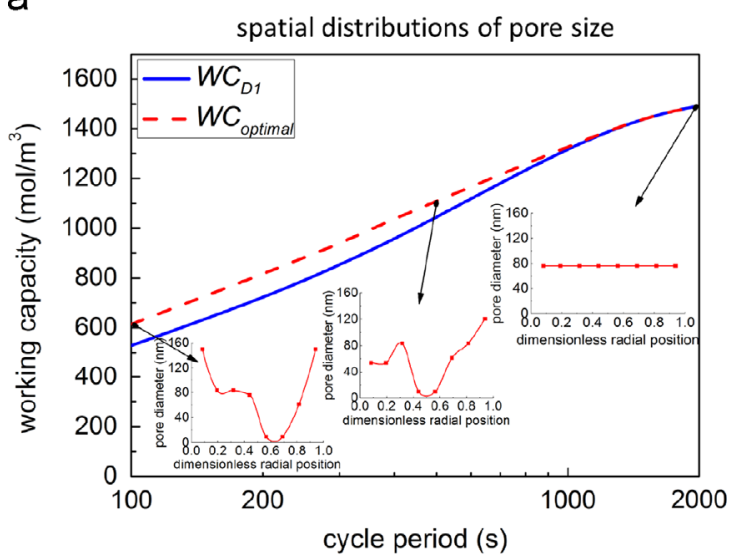

b

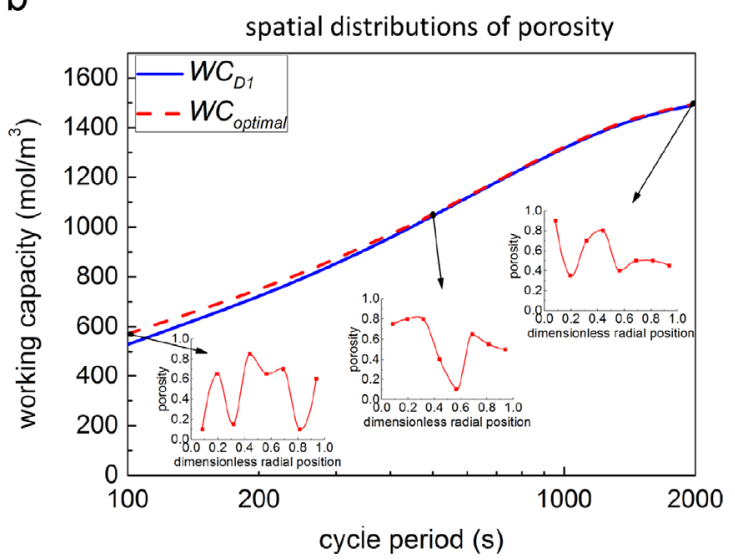

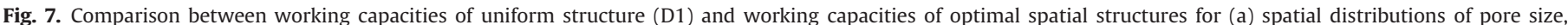

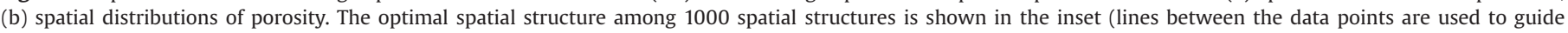
the eyes).

Therefore, the result that the uniform structure (D1) is preferred remains valid, independent of the exact $\tau(\varepsilon)$ relation.

\subsection{Optimal porosity of the adsorbent}

To optimize the average porosity of the adsorbent with the uniform spatial structure D1, the working capacity as a function of the average porosity ranging from 0.1 to 0.9 is presented in Fig. 9. A larger porosity not only leads to a larger diffusivity, but also to a smaller amount of material available for adsorption, implying that there is an optimal porosity, as confirmed by Fig. 9.

The optimal average porosity of the adsorbent with uniform structure is in the range of 0.4-0.6 when the perturbation cycle period is between $100 \mathrm{~s}$ and $2000 \mathrm{~s}$ and the volume-averaged pore diameter $\left(d_{a}\right)$ between $10 \mathrm{~nm}$ and $150 \mathrm{~nm}$. A larger porosity is favored for adsorbents with smaller volume-averaged pore diameter operating under shorter cycle period, while a smaller porosity is preferred for adsorbents with a larger volumeaveraged pore diameter operating under longer cycle period. For 
a

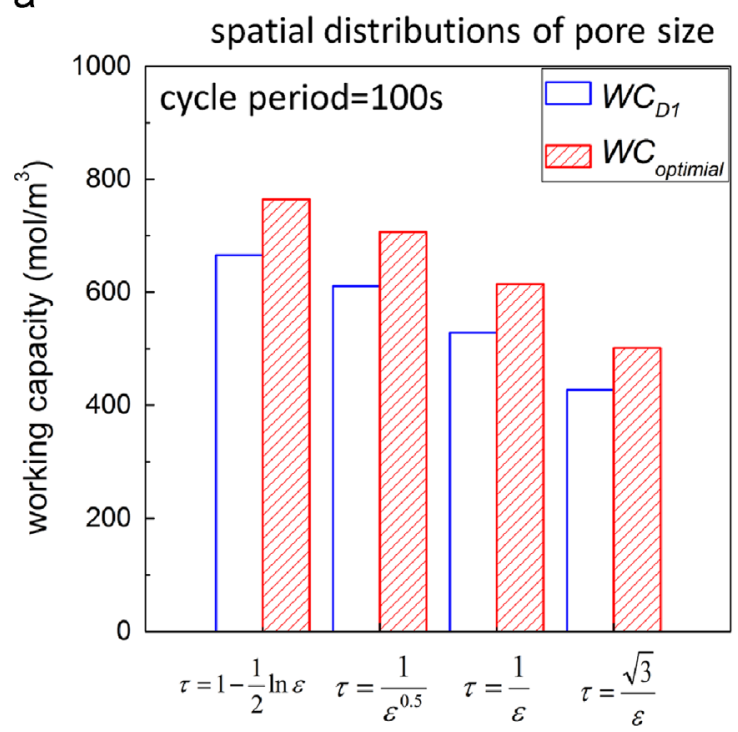

$b$

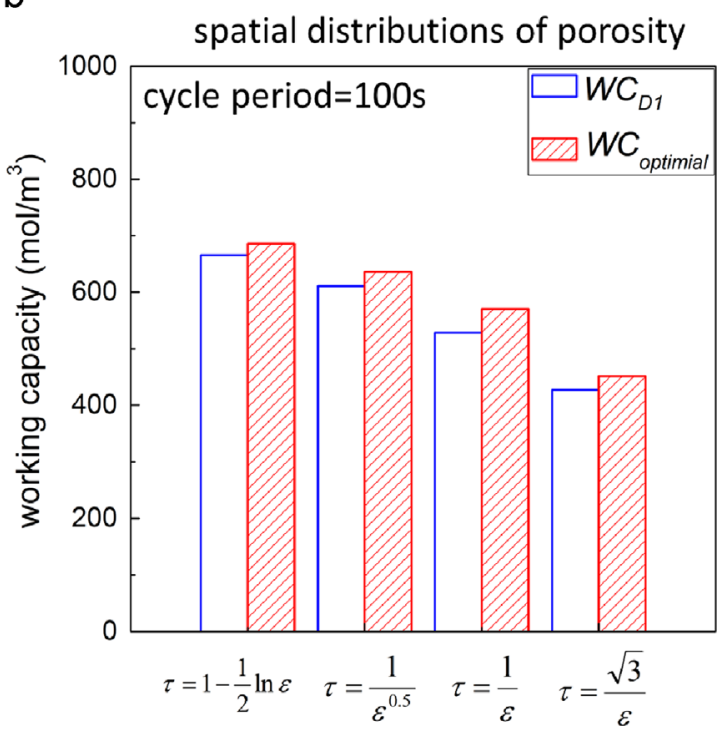

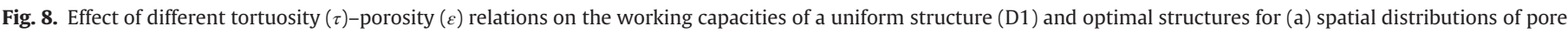
size and constant porosity and (b) spatial distributions of porosity and constant pore size. The square wave perturbation cycle period is $100 \mathrm{~s}$.

a

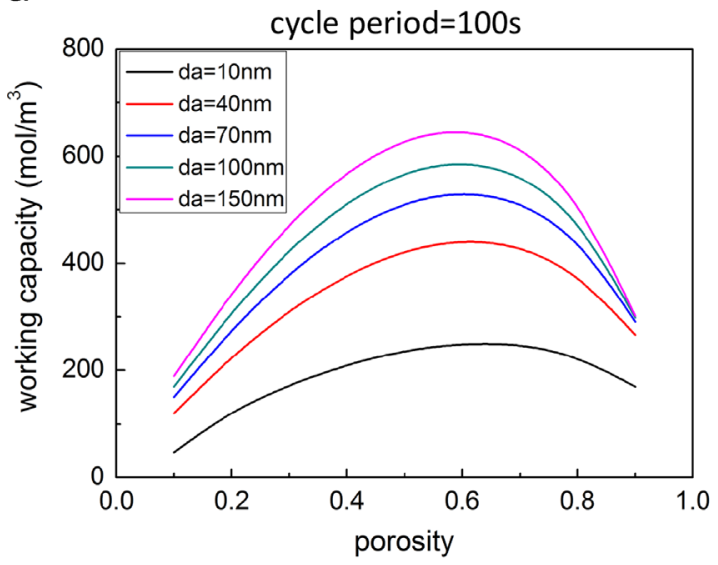

b

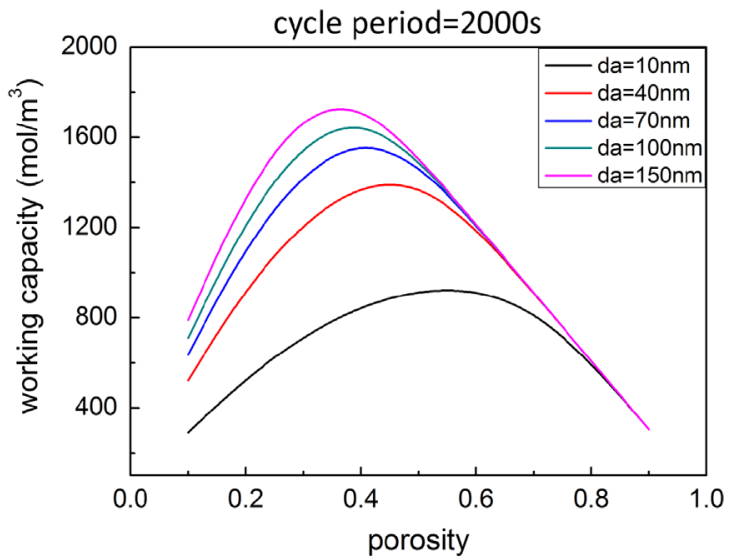

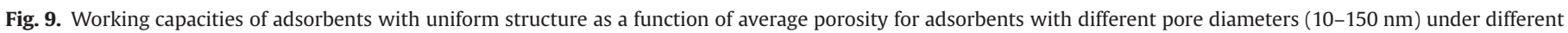
perturbation cycle periods, i.e., (a) $100 \mathrm{~s}$ and (b) $2000 \mathrm{~s}$.

example, the optimal average porosity is 0.6 when the volumeaveraged pore diameter is $10 \mathrm{~nm}$, while the optimal average porosity is 0.4 when the volume-averaged pore diameter is $150 \mathrm{~nm}$, as shown in Fig. 9b.

When the volume-averaged pore diameter is small and the cycle period is short, the dominant limitation in terms of increasing the working capacity is the diffusional resistance, rather than the amount of adsorbing material. Therefore, sacrificing some adsorbing material, which provides more space for diffusion, increases the working capacity. When the volume-averaged pore diameter is larger and the cycle period is longer, the rate of mass transfer is not important and a larger amount of adsorbing material is preferred to increase the working capacity.

In this article, the mass transfer rate is assumed to be controlled by bulk molecular diffusion and Knudsen diffusion. Provided that this assumption is valid when the volume-averaged pore diameter ranges from $10 \mathrm{~nm}$ to $150 \mathrm{~nm}$, the working capacities would rise with increasing volume-averaged pore diameter, as illustrated in Fig. 9. However, if the volume-averaged pore diameter is very large, the $5 \mathrm{~A}$ zeolite crystal would, correspondingly, also be large. In that case where large zeolite crystals are used - both diffusion limitations in the micropores and in the broad pores (mesopores and macropores) would be important, and the mass transfer rate could even be controlled by micropore diffusion. The latter is sub-optimal from the point of view of maximizing working capacity, so that further discussion is out of the scope of this paper.

\section{Conclusions}

In this work, the effect of different archetypical spatial structures on mass transfer in adsorbents was evaluated by using a continuum approach and employing a practically relevant evaluation criterion, namely the working capacity of adsorbents subjected to the square wave concentration perturbation with a wide range of cycle period. A uniformly distributed (D1) pore size and porosity is the preferred spatial structure, which is explained by comparing the loading profiles in the adsorbents. The generality of this result is further corroborated by comparing the uniform structure (D1) with 1000 randomly generated spatial structures, and using four empirical tortuosity-porosity relations. Finally, the optimal average porosity of the adsorbent with a uniform structure is found to be in the range 
of $0.4-0.6$ when the cycle period is between $100 \mathrm{~s}$ and $2000 \mathrm{~s}$, and the optimal average porosity is significantly affected by the volumeaveraged pore diameter and cycle period.

\section{Nomenclature}

$C_{i} \quad$ concentration of component $i$ in mesopores or macropores, $\mathrm{mol} / \mathrm{m}^{3}$

$C_{b, i} \quad$ concentration of component $i$ in bulk fluid, $\mathrm{mol} / \mathrm{m}^{3}$

$C_{\max , i}$ maximum concentration of component $i$ in bulk fluid, $\mathrm{mol} / \mathrm{m}^{3}$

$C_{\min , i}$ minimum concentration of component $i$ in bulk fluid, $\mathrm{mol} / \mathrm{m}^{3}$

d pore diameter, $\mathrm{nm}$

$d_{a} \quad$ volume-averaged pore diameter, $\mathrm{nm}$

$d_{H} \quad$ high limit of the pore diameter, $\mathrm{nm}$

$d_{L} \quad$ low limit of the pore diameter, $\mathrm{nm}$

$d_{n} \quad$ pore diameter of the ' $n$ th' zone, $n m$

$D_{e, i} \quad$ effective diffusivity of component $i, \mathrm{~m}^{2} / \mathrm{s}$

$D_{i, j} \quad$ binary molecular diffusivity of component $i$ in a mixture of $i$ and $j, \mathrm{~m}^{2} / \mathrm{s}$

$D_{k, i} \quad$ Knudsen diffusivity of component $i, \mathrm{~m}^{2} / \mathrm{s}$

$K \quad$ Henry coefficient, dimensionless

$M_{i} \quad$ molecular weight of component $i, \mathrm{~g} / \mathrm{mol}$

$M_{j} \quad$ molecular weight of component $j, \mathrm{~g} / \mathrm{mol}$

$N_{i} \quad$ flux of component $i, \mathrm{~mol} / \mathrm{m}^{2} / \mathrm{s}$

$l_{i} \quad$ volume-averaged loading of component $i, \mathrm{~mol} / \mathrm{m}^{3}$

$l_{\text {ads }, i} \quad$ volume-averaged loading of component $i$ at the end of adsorption period, $\mathrm{mol} / \mathrm{m}^{3}$

$l_{d e s, j} \quad$ volume-averaged loading of component $i$ at the end of desorption period, $\mathrm{mol} / \mathrm{m}^{3}$

total pressure, bar

$P$

$q_{i}$

$R$

radial position, $\mathrm{m}$

$R_{g} \quad$ ideal gas law constant, $\mathrm{J} / \mathrm{mol} / \mathrm{K}$

$R_{n} \quad$ radial position of the ' $n$ th' zone, $\mathrm{m}$

$R_{p} \quad$ radius of pellet, $\mathrm{m}$

$t$ time, s

$T \quad$ temperature, $\mathrm{K}$

$V_{i} \quad$ pore volume of the 'ith' zone, $\mathrm{m}^{3}$

$V_{p} \quad$ pore volume of the pellet, $\mathrm{m}^{3}$

Greek letters

$\begin{array}{ll}\sigma & \text { standard deviation, } \mathrm{nm} \\ \theta & \text { cycle period, s } \\ \Omega & \text { diffusion collision integral, dimensionless } \\ \varepsilon & \text { porosity of adsorbent, dimensionless } \\ \tau & \text { tortuosity, dimensionless }\end{array}$

Subscripts and superscripts

$\begin{array}{ll}a & \text { average } \\ b & \text { bulk } \\ i, j & i \text { or } j \text { component } \\ H & \text { high limit } \\ L & \text { low limit } \\ n & \text { ' } n \text { th' zone } \\ p & \text { pellet } \\ \text { ads } & \text { adsorption } \\ \text { des } & \text { desorption } \\ \text { max } & \text { maximum } \\ \text { min } & \text { minimum }\end{array}$

optimal optimal

\section{Acknowledgments}

X.G. is supported by the National Basic Research Program of China (2012CB720501), and by the National Natural Science Foundation of China (21376076). G.Y. is supported by the China Scholarship Council (CSC) for the research at UCL, and M.-O.C. is supported by the UK's EPSRC “Frontier Engineering” Centre for Nature Inspired Engineering.

\section{Appendix A. Supporting information}

Supporting data associated with this article can be found in the online version at http://dx.doi.org/10.1016/j.ces.2015.04.024.

\section{References}

Bárcia, P.S., Silva, J.A.C., Rodrigues, A.E., 2005. Adsorption equilibrium and kinetics of branched hexane isomers in pellets of BETA zeolite. Microporous Mesoporous Mater. 79, 145-163.

Beeckmann, J.W., Froment, G.F., 1980. Catalyst deactivation by site coverage and pore blockage. finite rate of growth of the carbonaceous deposit. Chem. Eng. Sci. 35, 805-812.

Bruggeman, D.A.G., 1935. Berechnung verschiedener physicalischer konstate von heterogen substanzen. Ann. Phys. 24 (5), 636-664.

Carman, P.C., 1937. Fluid flow through granular beds. Trans. Inst. Chem. Eng. 17 150-166.

Carta, G., Rodrigues, A.E., 1993. Diffusion and convection in chromatographic processes using permeable supports with a bidisperse pore structure. Chem. Eng. Sci. 48 (23), 3927-3935.

Da Silva, F.A., Rodrigues, A.E., 1999. Adsorption equilibria and kinetics for propylene and propane over $13 \mathrm{X}$ and $4 \mathrm{~A}$ zeolite pellets. Ind. Eng. Chem. Res. 38 2051-2057.

Foster, R.N., Butt, J.B.A., 1966. Computational model for the structure of porous materials employed in catalysis. AIChE J. 12, 180-185.

Gheorghiu, S., Coppens, M-O., 2004. Optimal bimodal pore networks for heterogeneous catalysis. AIChE J. 50, 812-820.

Hollewand, M.P., Gladden, L.F., 1992. Modeling of diffusion and reaction in porous catalysts using a three-dimensional network model. Chem. Eng. Sci. 47 $1761-1770$.

Johannessen, E., Wang, G., Coppens, M.-O., 2007. Optimal distributor networks in porous catalyst pellets. I. Molecular diffusion. Ind. Eng. Chem. Res. 46, 4245-4256.

Johnson, M.F., Stewart, W.E., 1965. Pore structure and gaseous diffusion in solid catalysts. J. Catal. 4, 248-252.

Keil, F.J., 1999. Diffusion and reaction in porous networks. Catal. Today 53, 245-258.

Kočí, P., Novák, V., Štěpánek, F., Marek, M., Kubíček, M., 2010. Multi-scale modelling of reaction and transport in porous catalyst. Chem. Eng. Sci. 65, 412-419.

Kočí, P., Štěpánek, F., Kubíček, M., Marek, M., 2006. Meso-scale modelling of CO oxidation in digitally reconstructed porous $\mathrm{Pt} / \gamma-\mathrm{Al}_{2} \mathrm{O}_{3}$ catalyst. Chem. Eng. Sci. 61, 3240-3249.

Krishna, R., Wesselingh, J.A., 1997. The Maxwell-Stefan approach to mass transfer. Chem. Eng. Sci. 52, 861-911.

Lu, Z.P., Loureiro, J.M., Le Van, M.D., Rodrigues, A.E., 1993. Pressure swing adsorption processes: intraparticle diffusion/convection models. Ind. Eng. Chem. Res. 32 (11), 2740-2751.

Mann, R., Thomson, G., 1987. Deactivation of a supported zeolite catalyst: simulation of diffusion, reaction and coke deposition in a parallel bundle. Chem. Eng. Sci. 42, 555-563.

Novák, V., Štěpánek, F., Kočí, P., Marek, M., Kubíček, M., 2010. Evaluation of local pore sizes and transport properties in porous catalysts. Chem. Eng. Sci. 65, 2352-2360.

Novák, V., Kočí, P., Gregor, T., Choi, J-S., Štěpánek, F., Marek, M., 2013. Effect of cavities and cracks on diffusivity in coated catalyst layer. Catal. Today 216 $142-149$.

Novák, V., Ortel, E., Winter, B., Butz, B., Paul, B., Kočí, P., Marek, M., Spiecker, E., Kraehnert, R., 2014. Prototyping of catalyst pore-systems by a combined synthetic, analytical and computational approach: application to mesoporous $\mathrm{TiO}_{2}$. Chem. Eng. J. 248, 49-62.

Petkovich, N.D., Stein, A., 2013. Controlling macro- and mesostructures with hierarchical porosity through combined hard and soft templating. Chem. Soc Rev. 42, 3721-3739.

Poling, B.E., Prausnitz, J.M., O'Connell, J.P., 2001. The Properties of Gases and Liquids 5th ed. McGraw-Hill, Boston, MA.

Rao, S.M., Coppens, M.-O., 2010. Mitigating deactivation effects through rationa design of hierarchically structured catalysts: application to hydrodemetalation. Ind. Eng. Chem. Res. 49, 11087-11097.

Rao, S.M., Coppens, M.-O., 2012. Increasing robustness against deactivation of nanoporous catalysts by introducing an optimized hierarchical pore networkapplication to hydrodemetalation. Chem. Eng. Sci. 83, 66-76. 
Rezaei, F., Webley, P.A., 2012. Optimal design of engineered gas adsorbents: porescale level. Chem. Eng. Sci. 69, 270-278.

Rodrigues, A.E., Ahn, B.J., Zoulalian, A., 1982. Intraparticle-forced convection effect in catalyst diffusivity measurements and reactor design. AIChE J. 28 (4), 541-546.

Rodrigues, A.E., Lu, Z.P., Loureiro, J.M., 1991. Residence time distribution of inert and linearly adsorbed species in fixed-bed containing "large-pore" supports: applications in separation engineering. Chem. Eng. Sci. 46 (11), 2765-2773.

Ruthven, D., 1984. Principles of Adsorption and Adsorption Processes. WileyInterscience, New York.

Sahimi, M., Gavalas, G.R., Tsotsis, T.T., 1990. Statistical and continuum models of fluid-solid reactions in porous media. Chem. Eng. Sci. 45, 1443-1502.

Seda, L., Zubov, A., Bobak, M., Kosek, J., Kantzas, A., 2008. Transport and reaction characteristics of reconstructed polyolefin particles. Macromol. React. Eng. 2 495-512.

Silva, J.A.C., Rodrigues, A.E., 1997a. Equilibrium and kinetics of n-hexane sorption in pellets of 5A zeolite. AIChE J. 43, 2524-2534.

Silva, J.A.C., Rodrigues, A.E., 1997b. Fixed-bed adsorption of n-pentane/isopentane mixtures in pellets. Ind. Eng. Chem. Res. 36, 3769-3777.

Solsvik, S., Jakobsen, H.A., 2011. Modeling of multicomponent mass diffusion in porous spherical pellets: application to steam methane reforming and methanol synthesis. Chem. Eng. Sci. 66, 1986-2000.

Szekely, J., Evans, J.W.A., 1970. Structural model for gas-solid reactions with a moving boundary. Chem. Eng. Sci. 25, 1091-1107.

Triantafillidis, C., Elsaesser, M.S., Hu, N., 2013. Chemical phase separation strategies towards silica monoliths with hierarchical porosity. Chem. Soc. Rev. 42, 3833-3846.

Václavík, M., Dudák, M., Novák, V., Medlí, R., Štěpánek, F., Marek, M., Kočí, P., 2014 Yeast cells as macropore bio-templates enhancing transport properties and conversions in coated catalyst layers for exhaust gas oxidation. Chem. Eng. Sci. $116,342-349$.
Voronoi, G., 1908. Novel applications of continuous parameters on the theory of quadratic forms. J. Reine Angew. Math. 134, 198-287.

Wakao, N., Smith, J.M., 1964. Diffusion and reaction in porous catalysts. Ind. Eng. Chem. Fundam 3, 123-127.

Wakao, N., Smith, J.M., 1962. Diffusion in catalyst pellets. Chem. Eng. Sci. 17, 825-834.

Wang, G., Coppens, M.-O., 2008. calculation of the optimal macropore size in nanoporous catalysts and its application to DeNOx catalysis. Ind. Eng. Chem. Res. 47, 3847-3855.

Wang, G., Coppens, M.-O., 2010. Rational design of hierarchically structured porous catalysts for autothermal reforming of methane. Chem. Eng. Sci. 65, 2344-2351.

Wang, G., Johannessen, E., Kleijn, C.R., de Leeuw, S.W., Coppens, M.-O., 2007. Optimizing transport in nanostructured catalysts: a computational study. Chem. Eng. Sci. 62, 5110-5116.

Weissberg, H.L., 1963. Effective diffusion coefficient in porous media. J. Appl. Phys. 34, 2636-2639.

Weisz, P.B., Schwartz, A.B., 1962. Diffusivity of porous oxide-gel derived catalyst particles. J. Catal. 1, 399-406.

Welty, J.R., Wicks, C.E., Wilson, R.E., Rorrer, G.L., 2008. Fundamentals of Momentum, Heat and Mass Transfer, 5th ed. John Wiley and Sons, Hoboken.

Wheeler, A., 1951. Reaction rates and selectivity in catalyst pores. Adv. Catal. 3, 249-327.

Winterfeld, P.H., Scriven, L.E., Davis, H.T., 1981. Percolation and conductivity of random two-dimensional composites. J. Phys. Chem. 14, 2361-2376.

Yao, J., Huang, Y., Wang, H., 2010. Controlling zeolite structures and morphologies using polymer networks. J. Mater. Chem. 20, 9827-9831.

Ye, G., Duan, X., Sui, Z., Zhu, K., Zhou, X., Yuan, W., 2014. Evaluation of approximations for concentration-dependent micropore diffusion in sorbent with bidisperse pore structure. Adsorption 20, 843-853.

Zhang, L., Seaton, N.A., 1992. Prediction of the effective diffusivity in pore networks close to a percolation threshold. AIChE J. 38 (11), 1816-1824. 